\title{
Líquenes de Pueblo Libre, una localidad andina en la Cordillera Negra (Huaylas, Ancash, Perú)
}

\section{Lichens of Pueblo Libre, an Andean locality from Cordillera Negra (Huaylas, Ancash, Perú)}

Ángel Ramírez ${ }^{1}$ A Asunción Cano ${ }^{1,2}$

Presentado: $16 / 06 / 2005$

Aceptado: $\quad 08 / 11 / 2005$

\section{Resumen}

El presente trabajo informa los resultados del estudio de la liquenobiota de la localidad andina de

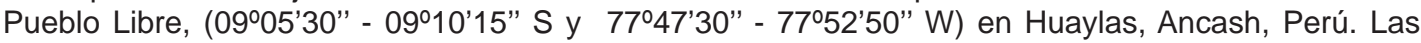
colectas se realizaron desde los 2200 hasta los $4450 \mathrm{~m}$ de altitud. El presente trabajo da a conocer 38 especies, agrupadas en 29 géneros y 17 familias y además del grupo imperfecto. Se presentan claves para familias, géneros y especies. Para cada especie se presenta una descripción macroscópica y microscópica y de reacción química, asimismo se menciona el hábitat y material examinado.

Palabra clave: Líquenes, Biodiversidad, Andes, Ancash, Perú.

\section{Abstract}

In this paper we report the lichens of Pueblo Libre, an Andean locality, (0905'30" - 0910'15" S and 77ㄴㄱ'30" - 77-52'50" W) in Huaylas, Ancash, Peru. Surveys were made at 2200 to 4450 m of elevation. In all 38 species, 29 genera and 17 families, besides one imperfect taxon are are reported. A key of family, genera and species is included. Morphological and anatomical features are described for each species, including its chemical reaction, habitat and vouchers.

Keywords: Lichen, Biodiversity, Andes, Ancash, Peru.

\section{Introducción}

Las definiciones modernas consideran a los líquenes como hongos liquenizados. Constituyen una asociación entre un hongo o micobionte y un simbionte fotosintético o fotobionte, de cuya interacción se origina un talo estable, con estructura y fisiología específicas (Barreno, 1998).

Los líquenes son pioneros en la colonización de rocas, desintegrándolas para la formación de suelo, permitiendo el crecimiento de diversos tipos de vegetación rupícola o saxícola, musgos y ciertas plantas vasculares (Herrera \& Ulloa, 1990), jugando así un papel importante en el ciclo de la materia en los ecosistemas. Los líquenes tienen una amplia distribución, se encuentran desde los polos

\footnotetext{
${ }^{1}$ Museo de Historia Natural, Universidad Nacional Mayor de San Marcos. Avda. Arenales 1256, Lima. Apartado 14-0434, Lima 14, Perú. Correo-e Ángel Ramírez: liquenesperu@yahoo.com

${ }^{2}$ Instituto de Investigación de Ciencias Biológicas «Antonio Raimondi»- Facultad de Ciencias

Biológicas, UNMSM.
}

hasta el Ecuador; desde el nivel del mar hasta los picos altos de las montañas y desde los sitios más húmedos como las selvas y los bosques hasta las zonas desérticas (Herrera \& Ulloa, 1990). Los líquenes pueden ser utilizados como bioindicadores de la calidad del aire (Brodo et al., 2001).

Los estudios taxonómicos son complejos, e incluyen el estudio de los caracteres macroscópicos, microscópicos, químicos (K, C, KC, Pd, I y UV), moleculares (TLC, HPLC) y genéticos (secuencias de $\mathrm{ADN}$ ). Asimismo se requiere estudiar su distribución a nivel mundial.

El objetivo de esta investigación fue realizar un estudio de la líquenobiota del distrito de Pueblo Libre (Huaylas, Ancash). Para lo cual se describe caracteres macroscópicos, microscópicos y algunos químicos.

\section{Área de estudio}

El área de estudio está situada en el distrito de Pueblo Libre, provincia de Huaylas, departamento de Ancash; en la margen izquier- 
da del río Santa. Comprendió 13 localidades (Fig. 1), entre los 2200 hasta los $4450 \mathrm{~m}$ de altitud y entre las coordenadas geográficas de $09^{\circ} 05^{\prime} 30^{\prime \prime}$ a $09^{\circ} 10^{\prime} 15^{\prime \prime} \mathrm{S}$ y $77^{\circ} 47^{\prime} 30^{\prime \prime}$ a $77^{\circ} 52^{\prime} 50^{\prime \prime} \mathrm{W}$.

En esta área se pueden encontrar tres tipos de climas: el de las Vertientes Occidentales, de clima seco y sin lluvias durante el invierno; el de Puna, frío y húmedo debido a la altitud y a la continentalidad; y el de los Valles Interandinos, de clima templado con inviernos fríos y con características locales de semiaridez (Ferreyra, 1986).

$\mathrm{El}$ área de estudio presenta los siguientes tipos de vegetación: el matorral, que ocurre en el fondo de los valles y las laderas hasta cerca de los $3800 \mathrm{~m}$, caracterizado por la presencia de arbustos como Flourencia macrophylla, Dodonaea viscosa, Gochnatia patazina y Mimosa spp; el pajonal de puna, por encima de los $3800 \mathrm{~m}$, donde se encuen- tran variadas gramíneas como Jarava ichu, Calamagrostis spp. y Muhlenbergia spp. y el rodal de Puya, destacando principalmente la Puya raimondii la cual alterna con arbustos dispersos de Baccharis spp.

\section{Colecta}

Las colectas se realizaron entre los años 2000 y 2002 y consistieron en muestreos ad libitum en cada lugar tanto sobre roca, suelo, musgo o corteza de arbustos y árboles, de preferencia muestras con estructuras fértiles. Las muestras se coloca en bolsas de papel, con información de número de colecta, biotipo, color y sustrato, localidad, fecha, coordenadas geográficas y altitud.

En el laboratorio las muestras se colocaron en sobres de papel bulki revestidos con papel kraft, y fueron secadas exponiéndolas al sol, para que no pierdan la tonalidad del talo o sufrir desintegracion de las sustancias liquénicas
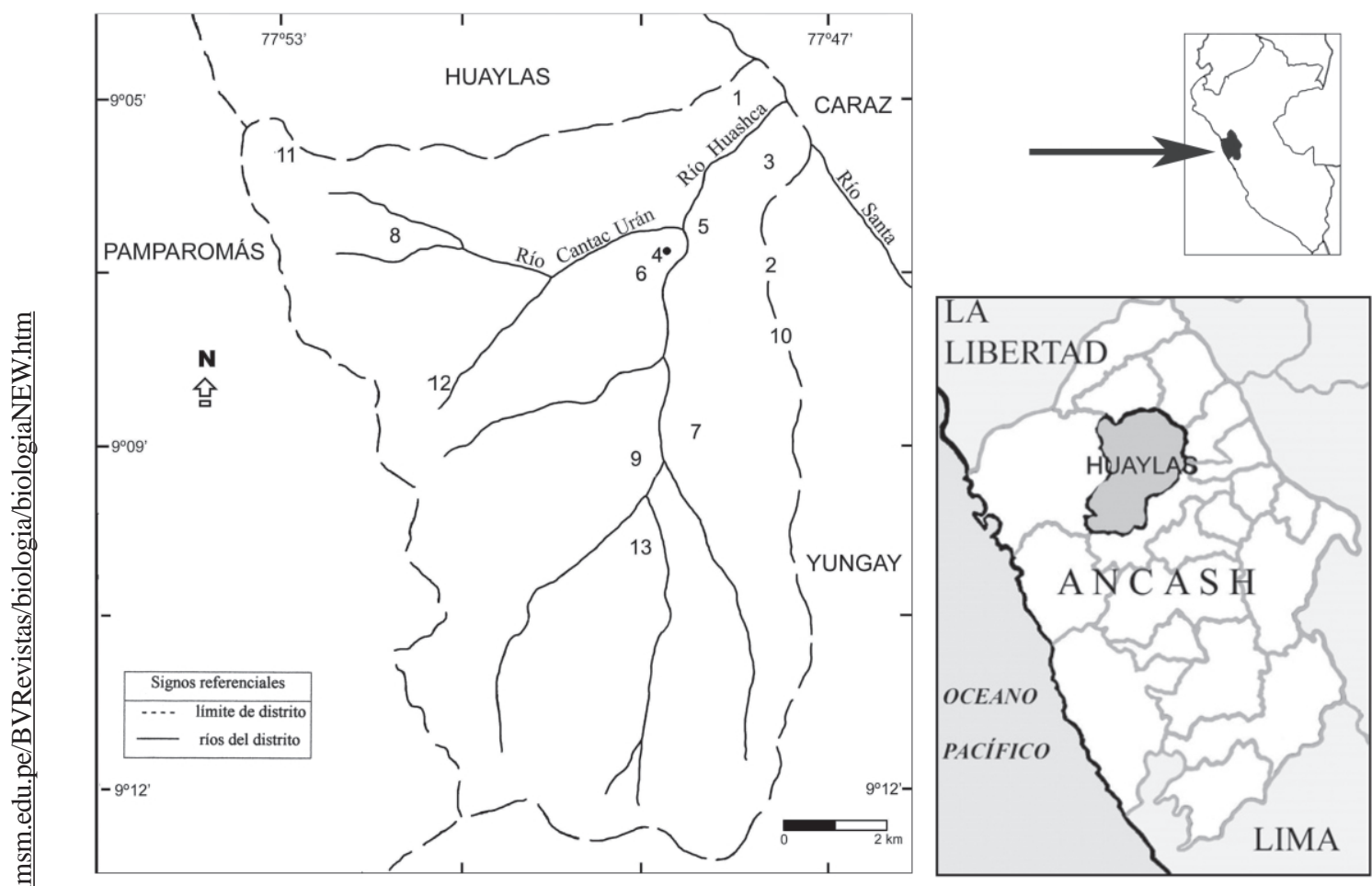

Figura 1. Localidades muestreadas del distrito de Pueblo Libre. 1 Tocash, 2. Cerro Yanaico, 3. Huaracayoc, 4. Pueblo Libre (Capital), 5. Ángüel Pampa, 6. Cerro Huantar, 7. Hoyada, 8. Coiroqsho, 9. Marca, 10. Cerro Nuna Riqoq, 11. Huashta Cruz, 12. Coracayoc y 13. Antircán. 
(Coutino, 1986). Todas las colecciones fueron depositadas en el Herbario San Marcos (USM).

\section{Características estudiadas}

Se tomaron en cuenta los caracteres macroscópicos, microscópicos, el test de coloración con potasio $(\mathrm{K})$, y de manera particular el uso de hipoclorito de calcio (C) y parafenilendiamina (PD).

Características macroscópicas. Son la clase de biotipo; el color del talo, presencia de isidios, papilas, cilios y rizinas; presencia o ausencia de cuerpos fructíferos. En los apotecios se describen la forma, el tamaño, el color y la posición en el talo.

Características microscópicas. En el talo son la presencia o ausencia de córtex y el tipo de fotobionte; en el ascoma son tipo de asca, número de ascosporas por asca, forma, tamaño, septación y color para la ascospora, y en la paráfisis son la septación y ramificación.

Examen de coloración con $\mathbf{K}$. Proporciona coloración positiva púrpura o amarilla; con $\mathrm{C}$ es positiva si da color rojo y con PD es positiva si da color anaranjado. En estas pruebas de coloración la reacción es negativa si no presenta el color mencionado.

\section{Determinación de los taxa}

Se emplearon claves y descripciones disponibles en la literatura especializada (Bruce, 1960; Sato, 1968; Hale, 1979; Ahti, 1984; Galloway, 1985; Walker 1985; Kashiwadani, 1987; Parmastro, 1987; Moberg, 1990; Almborn, 1992; Elix \& Nash, 1992; Moberg, 1993; Elix, 1994; Galloway, 1994; Vitikainen, 1998; Sipman, 1999). También fueron revisados los ejemplares de líquenes depositados en los Herbario San Marcos (USM), Herbarium Truxillense (HUT) y Herbario Weberbauer (MOL); asimismo se consultó a especialistas extranjeros.

\section{Resultados}

En la liquenobiota del distrito de Pueblo Libre, Huaylas, Ancash, se registran un total de 38 especies, agrupadas en 29 géneros y al Grupo Imperfecto.

\section{Clave para diferenciar las familias de lí- quenes del distrito de Pueblo Libre.}

1a. Talo crustáceo 2

1b. Taloescamoso, folioso, fructicoso odimórfico 3

2a. Apotecio irregular. 4

2b. Apotecio circular.

Arthoniaceae

4a. Ascospora con un septo.

Rhizocarpaceae

4b. Ascospora con muchos septos

Lecideaceae

5a. Apotecio lecideíno

6

5b. Apotecio lecanorino

6a. Asca con 8 ascosporas

6b. Asca con numerosas ascosporas (64)

Acarosporaceae

7a. Ascospora sin septo $\quad 8$

7b. Ascospora con septo o lóculos

8a. Talo verde amarillo, ascospora de $8 \mu \mathrm{m}$ de largo

Lecanoraceae

8b. Talo marrón, ascospora de $25 \mu \mathrm{m}$ de largo

Pertusariaceae

9a. Ascospora con un septo

Physciaceae

9b.Ascospora polarilocular

Teloschistaceae

3a. Talo escamoso

Psoraceae

3b. Talo folioso, fructicoso o dimórfico

10

10a. Talo folioso

10b. Talo fructicoso o dimórfico

11a. Talo de color amarillo

Candelariaceae

11b. Talo de color no amarillo

13a. Talo con cianobionte

13b. Talo con clorobionte

14a. Talo de color blanco

Thelephoraceae

14b. Talo de color marrón

15a. Apotecio lecideíno

Umbilicariaceae

15b. Apotecio lecanorino

16a. Talo sin cifelas

16b. Talo con cifelas

Peltigeraceae

Lobariaceae

17a. Ascospora sin septo

Parmeliaceae

17b. Ascospora con un septo 
12a. Talo fructicoso

12b. Talo dimórfico

Cladoniaceae

18a. Talo de color amarillo a verde

19

18b. Talo de color blanco

Grupo Imperfecto

19a. Ascospora sin septo

Parmeliaceae

19b. Ascospora con septo

Ramalinaceae

\section{Descripción de las especies}

FAmilia Acarosporaceae Zahlbr.

\section{Acarospora schleicheri (Ach.) Mass.}

Liquen crustáceo areolado, amarillo claro; terrícola. Apotecio circular, cóncavo, inmerso en el talo y diámetro de $0,4 \mathrm{~mm}$, con borde talino y epitecio amarillo. Talo con córtex superior, heterómero con clorobionte Protococcus sp.; apotecio lecanorino; asca con 64 ascosporas elipsoidales, con septos, hialinas y de 2,88 x 1,44 $\mu \mathrm{m}$; paráfisis con septos y sin ramificación. Talo $\mathrm{K}-$. Habita en suelo arcilloso con matorral. (Fig. 2a)

M.E.: Cerro Nuna Riqoq, 3400 m, 17 may. 00, A. Ramírez \& A. Cano 224 (USM); Huashta Cruz, 3400 m, 26 may. 01, A. Ramírez \& A. Cano 497 (USM).

\section{Familia Arthoniaceae Reichenb.}

\section{Clave para diferenciar las especies del género Arthonia}

1a. Talo de color gris, ascospora con células iguales. Arthonia lapidicola

1b. Talo de color marrón, ascospora con células desiguales. Arthonia rupicola

\section{Arthonia lapidicola (Tayl.) Branth \& Rostr.}

Liquen crustáceo, gris, con apotecios negros; saxícola. Apotecio irregular, plano, sésil y diámetro de 0,4 a 0,6 mm, sin borde talino y con epitecio negro. Talo con córtex superior, heterómero con clorobionte Trentepohlia sp. y Palmella sp. Asca con ocho ascosporas elipsoidales con un septo trasversal, células iguales, marrones y de 16,3 x 7,7 $\mu \mathrm{m}$; paráfisis con septo y sin ramificación. Talo K-. Habita en ladera rocosa con pajonal de puna.

M.E.: Huashta Cruz, 4000-4200 m, 16 may. 00, A. Ramírez \& A. Cano 251 (USM).

\section{Arthonia rupicola Fink}

Liquen crustáceo, marrón claro, con apotecios negros; saxícola. Apotecio irregular, plano, sésil y diámetro de $0,3 \mathrm{~mm}$, sin borde talino y con epitecio negro. Talo con córtex superior, heterómero con clorobionte Trentepohlia sp. y Palmella sp. Asca con 8 ascosporas elipsoidales con un septo transversal, células diferentes, marrones y de 12,4 x 6,7 $\mu \mathrm{m}$; paráfisis con septo y sin ramificación. Talo K-. Habita en ladera pedregosa con matorral.

M.E.: Huashta Cruz, 3500 m, 13 abr. 01, A. Ramírez \& A. Cano 441,426 (USM).

Familia Candelariaceae Hakulinen

\section{Clave para diferenciar las especies de la familia Candelariaceae}

1a.Talo sin ombligo

Candelaria concolor

1b.Talo con ombligo Placomaronea candelaroides

\section{Candelaria concolor (Dickson) Arnold}

Liquen folioso, amarillo; cortícola. Talo con rizinas. Apotecio circular, cóncavo, sésil, diámetro de 0,7 a $0,8 \mathrm{~mm}$, con borde talino y epitecio amarillo. Talo con córtex superior e inferior, heterómero con clorobionte Protococcus sp. Apotecio lecanorino; asca con 16 ascosporas elipsoidales sin septos, con dos lóculos, hialina y de 9,2 × 3,2 $\mu \mathrm{m}$; paráfisis con septos y ramificación. Talo K-. Habita en ladera pedregosa con matorral.

M.E.: Cerro Yanaico, 2400 - 300, 01 nov. 00, A. Ramírez \& A. Cano 375 (USM); Hoyada, 2800 - 3000 m, 07 oct. 01, A. Ramírez \& A. Cano 738 (USM). 


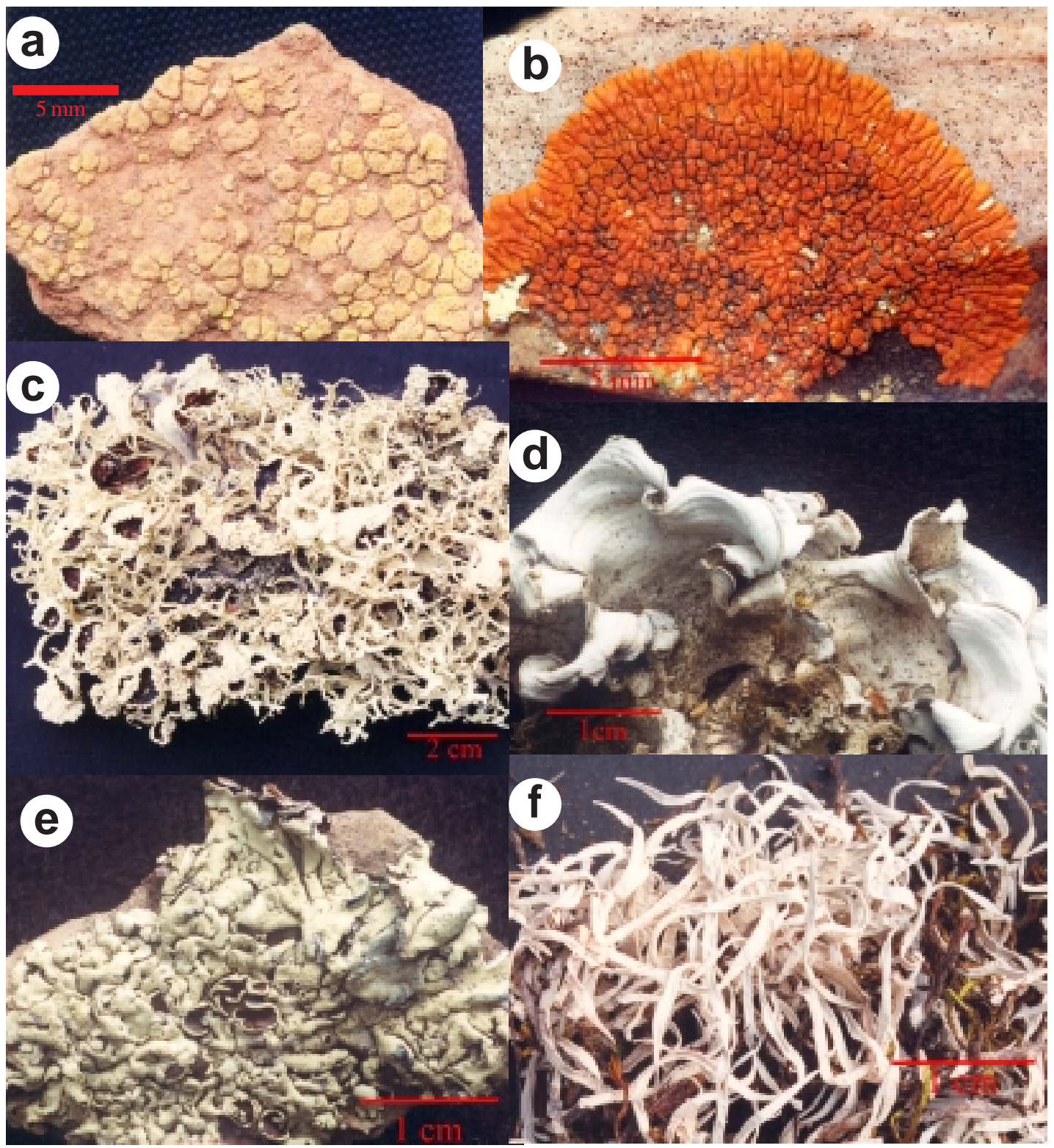

Figura 2. (a) Acarospora schleicheri, (b) Caloplaca saxicola, (c) Everniopsis trulla, (d) Dictyonema glabratum, (e) Psiloparmelia distincta y (f) Thamnolia vermicularis.

\section{Placomaronea candelaroides Räs.}

Liquen folioso con ombligo; mostaza; saxícola. Apotecio circular, convexo, sésil, diámetro de $0,8 \mathrm{~mm}$, con borde talino y epitecio amarillo. Talo con córtex, heterómero con clorobionte Trebouxia sp. Apotecio lecanorino; asca con 16 ascosporas elipsoidales sin septo, bilocular, hialina y de 9,84 × 3,84 $\mu$ m; paráfisis con septo y ramificación. Talo K-. Habita en roquedales. (Fig. 3 a)

M.E.: Cerro Huantar, 2800 m, 09 oct. 99, A. Ramírez \& A. Cano 8 (USM); Coiroqsho, 2900 -3200, 08 oct. 01, A. Ramírez \& A. Cano 81 (USM); Hoyada, 2800-3000, 07 oct.01, A. Ramírez \& A. Cano 748 (USM).

Determinado: Dr. Sipman H. 
Familia Cladoniaceae Zenker

\section{Cladonia chlorophaea (Florke ex Somm.) Spreng.}

Liquen dimórfico, oliva claro; terrícola. Talo primario escamoso y el talo secundario (podecio) fructicoso. Podecio de $4 \mathrm{~mm}$ de alto y 1 a $2 \mathrm{~mm}$ de grosor, presentando copas y ausencia de apotecios. Talo primario con córtex, heterómero con clorobionte Pleurococcus sp. Podecio con soredios granulares y córtex, heterómero con clorobionte Pleurococcus sp. (Bruce 1960), con hifas perpendiculares a la superficie y hueco. Talo K-. Habita en laderas con suelo desnudo y matorral.

M.E.: Huashta Cruz, 3400 m, 26 may. 01, A. Ramírez \& A. Cano 489 (USM).

\section{FAMilia LeCANORACEAE KÖRBER}

\section{Lecanora garovaglii (Körber) Zahlbr.}

Liquen crustáceo, verde amarillo; saxícola. Apotecio circular, ligeramente convexo, sésil, diámetro de 0,7 a $1 \mathrm{~mm}$, con borde talino y epitecio marrón. Talo con córtex superior, heterómero con clorobionte Protococcus sp. Apotecio lecanorino; asca con ocho ascosporas elipsoidales, hialina y de $8,3 \times 5,2 \mu \mathrm{m}$; paráfisis con septo y sin ramificación. Talo K-. Habita en laderas pedregosas. (Fig. 3c)

M.E.: Huashta Cruz, 3400 m, 13 abr. 01, A. Ramírez \& A. Cano 438 (USM).

Determido: Dr. Thorsten L.

Familia Lecideaceae Chev.

Clave para diferenciar las especies del género Lecidea

1a. Liquen terrícola

Lecidea uliginosa

1b. Liquen saxícola

2

2a. Apotecios agrupados, ascospora mayor de $10 \mu \mathrm{m}$ en longitud Lecidea vorticosa

2b. Apotecios no agrupados, ascospora menor de $10 \mu \mathrm{m}$ en longitud

Lecidea auriculata

\section{Lecidea auriculata T. Fries}

Liquen crustáceoareolado, gris; saxícola. Apotecio circular, convexo, sésil, diámetro de 1 a 2 mm, sin borde talino y epitecio negro. Talo con córtex superior, heterómero con clorobionte Protococcus sp. Apotecio lecideíno; asca con ocho ascosporas elipsoidales sin septo, hialinas y de 8,8 × $6,5 \mu \mathrm{m}$; paráfisis con septo y sin ramificación. Talo $\mathrm{K}-\mathrm{yC}-$ . Habita en laderas rocosas con pajonal de puna.

M.E.: Huashta Cruz, 4000 m, 29 oct. 00, A. Ramírez \& A. Cano 351 (USM).

\section{Lecidea uliginosa (Schrader) Ach.}

Liquen crustáceo areolado, marrón; terrícola. Apotecio circular, convexo, sésil, diámetro de 1 a $2 \mathrm{~mm}$, sin borde talino y epitecio negro. Talo con córtex superior, heterómero con clorobionte Protococcus sp. Apotecio lecideíno; asca con cuatro ascosporas elipsoidales sin septo, hialinas y de $9 \times 6 \mu \mathrm{m}$; paráfisis con septos y sin ramificación. Talo $\mathrm{K}-\mathrm{y} \mathrm{C}-$. Habita en laderas pedregosas.

M.E.: Huashta Cruz, 3400 m, 26 may. 01, A. Ramírez \& A. Cano 487 (USM).

\section{Lecidea vorticosa (Floerke) Koerb.}

Liquen crustáceo areolado, gris; saxícola. Apotecio circular, plano, sésil, diámetro de 0,3 a $0,9 \mathrm{~mm}$, con borde propio y epitecio negro. Talo con córtex superior, heterómero con clorobionte Protococcus sp. (Bruce, 1960). Apotecio lecideíno; asca con cuatro ascosporas elipsoidales, sin septo, hialina y de 10,7 x 5,8 $\mu \mathrm{m}$; paráfisis septada y no ramificada. Talo $\mathrm{K}-\mathrm{y} \mathrm{C}-$. Habita en laderas rocosas con matorral. (Fig. 3d)

M.E.: Huashta Cruz, 3600 m, 13 abr. 01, A. Ramírez \& A. Cano 433 (USM).

Familia Lobariaceae Chev.

\section{Sticta fuliginosa (Hoffm.) Ach.}

Liquen folioso, marrón; muscícola. Cara superior con isidios. Cara inferior del talo con cifelas y sin rizinas. Apotecio ausente. Talo con 


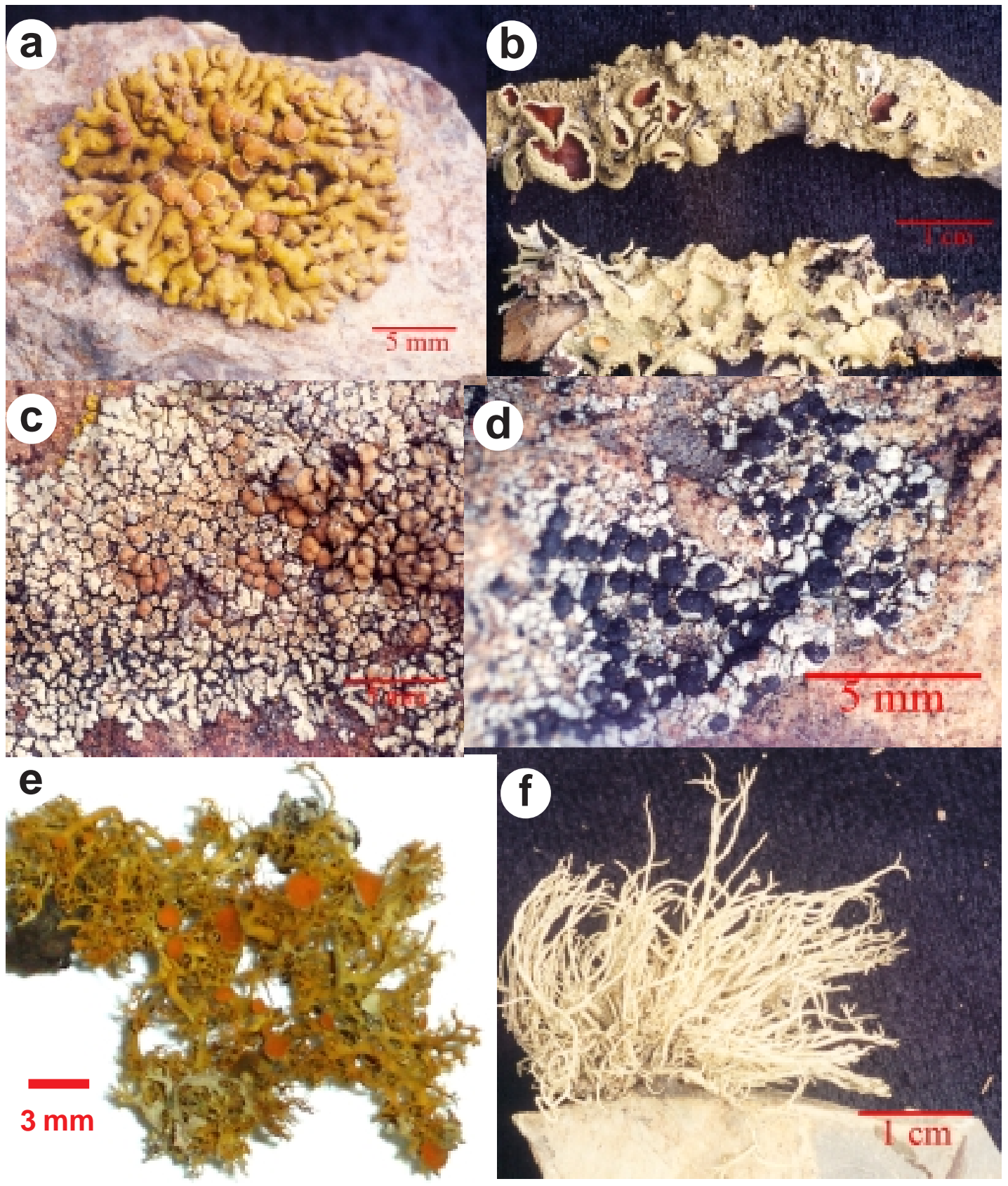

Figura 3. (a) Placomaronea candelaroides, (b) Flavoparmelia scabrosina, (c) Lecanora garovaglii, (d) Lecidea vorticosa, (e) Teloschistes chrysophthalmus y (f) Usnea durietzii.

córtex superior e inferior, heterómero con cianobionte Nostoc sp. (Galloway, 1985). Talo $\mathrm{K}-$. Habita en laderas con suelo arcilloso desnudo y matorral ralo.

M.E.: Coracayoc, 3400-3940 m, 05 ago. 01, A. Ramírez \& A. Cano 648 b (USM).
Familia Parmeliaceae ZenKer

Clave para diferenciar las especies de la familia Parmeliaceae

1a. Liquen folioso

1b. Liquen fructicoso

2a. Talo con cilios

Cetrariastrum $\mathrm{sp}$ 
2b. Talo sin cilos

4a. Liquen saxícola

4b. Liquen cortícola

5a. Lóbulos amplios

Psiloparmelia distincta

5b. Lóbulos estrechos Xanthoparmelia conspersa

6a. Talo de color gris

Pseudevernia sp.

6b. Talo de color verde oliva Flavoparmelia scabrosina

3a. Talo sin cordón central

Everniopsis trulla

3b. Talo con cordón central

7

7a. Liquen saxícola

Usnea durietzii

7b. Liquen cortícola

9

9a. Talo con soralio

Usnea sp.1

9b. Talo con papilas y fibrilas

Usnea sp.2

\section{Cetrariastrum sp.}

Liquen folioso, marrón oscuro; cortícola. Talo con cilios y sin rizinas. Apotecio circular, cortamente pedicelado, diámetro de $0,8 \mathrm{a} 3 \mathrm{~mm}$, con borde talino y epitecio marrón claro. Talo con córtex superior e inferior, heterómero con clorobionte Trebouxia sp. Apotecio lecanorino; asca con ocho ascosporas elipsoidales sin septo, hialina y de $6,4 \times 4,8 \mu \mathrm{m}$; paráfisis con septos y sin ramificación. Talo K-. Habita en laderas pedregosas con pajonal de puna.

M.E.: Huashta Cruz, 4000-4200 m, 16 may. 00, A. Ramírez \& A. Cano 250 (USM).

\section{Everniopsis trulla (Ach.) Nyl.}

Liquen fructicoso, verde amarillo claro; cortícola y creciendo entre rocas. Apotecio circular, cóncavo, cortamente pedicelado, diámetro de 5 a $15 \mathrm{~mm}$, con borde talino y epitecio marrón oscuro. Talo sólido, con córtex, heterómero con clorobionte Trebouxia sp. Apotecio lecanorino; asca con ocho ascosporas elipsoidales, sin septo, hialina y de 11 x $6 \mu \mathrm{m}$; paráfisis con septo y sin ramificación. Talo $\mathrm{K}-$ . Habita en roquedales, rodales de Puya y laderas con matorral. (Fig. 2c)

M.E.: Cerro Yanaico, 2400-2650 m, 12 abr. 01, A. Ramírez \&A. Cano 396 (USM); Coracayoc,
3400 - 3940 m, 05 ago.01, A. Ramírez \& A. Cano 622 (USM); Huashta Cruz, 4090 m, 16 may. 00, A. Ramírez \& A. Cano 251 (USM).

\section{Flavoparmelia scabrosina Elix \&} J. Johnst.

Liquen folioso, verde oliva; cortícola. Talo sin rizinas. Apotecio circular, cóncavo, sésil, diámetro de 2 a $7 \mathrm{~mm}$, con borde talino y epitecio marrón. Talo con córtex superior e inferior, heterómero con clorobionte Trebouxia sp. Apotecio lecanorino, asca con seis ascosporas elipsoidales sin septo, hialina y de 12 x 7,7 $\mu \mathrm{m}$; paráfisis con septo y sin ramificación. Talo K-. Médula KC-y PD-. Habita en laderas con matorral. (Fig. 3b)

M.E.: Cerro Yanaico, 2400-3000 m, 01 nov. 00, A. Ramírez \& A. Cano 370 (USM); Coracayoc, 3400-3940 m, 05 ago. 01, A. Ramírez \& A. Cano 619 (USM); Coroiqsho, 2910-3220m, 08 oct. 01, A. Ramírez \& A. Cano 790 (USM); Tocash, 2300 m, 09 oct. 01, A. Ramírez \& A. Cano 825 (USM); Huaracayoc, 2530 m, 09 oct 01, A. Ramírez 836 (USM).

\section{Pseudevernia sp.}

Liquen folioso, gris claro; cortícola. Talo sin rizinas. Apotecio circular, cóncavo, ligeramente pedicelado, diámetro de 1.5 a $4 \mathrm{~mm}$, borde talino y epitecio marrón oscuro. Talo con córtex superior e inferior, heterómero con clorobionte Trebouxia sp. Apotecio lecanorino; asca con ocho ascosporas elipsoidales sin septo, hialinas y 10,2 x 4,7 $\mu \mathrm{m}$; paráfisis con septo y $\sin$ ramificación. Talo K - . Habita en laderas con matorral.

M.E.: Cerro Yanaico, 2400 - 3000 m, 01 nov. 00, Ramírez 371 (USM); Huashta Cruz, 3400 m, 13 abr. 01, Ramírez 428b (USM).

\section{Psiloparmelia distincta (Nyl.) Hale}

Liquen folioso, verde claro; saxícola. Talo sin rizinas. Apotecio circular, cóncavo, cortamente pedicelado, diámetro 1,5 a $3 \mathrm{~mm}$, con borde talino y epitecio marrón. Talo con 
córtex superior e inferior, heterómero con clorobionte Trebouxia sp. Apotecio lecanorino; asca con cuatro ascosporas esféricas a elipsoidales sin septo, hialina y de 9,8 x $6,2 \mu \mathrm{m}$; paráfisis con septos y sin ramificación. Talo K-, córtex PD + y médula K-. Habita en laderas rocosas con matorral. Fig. 2e.

M.E.: Huashta Cruz, 3800 m, 13 abr.01, A. Ramírez \& A. Cano 423(USM).

\section{Usnea durietzii Motyka}

Liquen fructicoso, verde amarillo; saxícola. Apotecio ausente. Talo grueso con soredios, sin papilas, con base manchada, sólido, con córtex, heterómero con clorobionte Trebouxia sp. y con cordón central. Talo K-, médula K-y PD-. Habita en roquedales.

M.E.: Huashta Cruz, 4000-4200m, 16 may. 00, 249(USM); Antircán, 3450 m, 08 ago. 01, A. Ramírez \& A. Cano 674(USM). (Fig. 3f)

\section{Usnea sp.1}

Liquen fructicoso, amarillo claro; cortícola. Apotecio ausente. Talo delgado con soralio, sin papilas, sólido, con córtex, heterómero con clorobionte Trebouxia sp. y con cordón central. Talo K-. Hábitat: Pajonal de puna y ladera con matorral.

M.E.: Huashta Cruz, 4000 - 4200 m, 16 may.00, A. Ramírez \& A. Cano 215(USM).

\section{Usnea sp. 2}

Liquen fructicoso, verde amarillo; cortícola. Apotecio ausente. Talo grueso sin soralio, con papilas y fibrillas, sólido, con córtex, heterómero con clorobionte Trebouxia sp. y con cordón central. Talo K-. Habita en laderas con matorral.

M.E.: Huashta Cruz, 3400 m, 13 abr. 01, A. Ramírez \& A. Cano 431(USM).

\section{Xanthoparmelia conspersa (Ach.) Hale}

Liquen folioso, verde claro; saxícola. Talo sin rizinas. Apotecio circular, ligeramente cóncavo, sésil, $6 \mathrm{~mm}$. de diámetro, con borde talino y epitecio marrón oscuro. Talo con córtex superior e inferior, heterómero con clorobionte Trebouxia sp. Apotecio lecanorino; asca con cuatro ascosporas elipsoidales sin septo, hialina y de 7,7 x 4,8 $\mu \mathrm{m}$; paráfisis con septo y $\sin$ ramificación. Talo $\mathrm{K}-$. Habita en laderas rocosas con matorral.

M.E.: Huashta Cruz, 3400 m, 13 abr. 01, A. Ramírez \& A. Cano 433(USM).

Determinado: Dr. Sipman H.

Familia Peltigeraceae W. Watson

\section{Peltigera austroamericana Zahlbr}

Liquen folioso con márgenes ascendiendo, marrón; terrícola. Apotecio, circular, convéxo, sésil, diámetro de $6 \mathrm{~mm}$, sin borde talino y con epitecio marrón. Talo con córtex superior y sin córtex inferior, heterómero con cianobionte Nostoc sp. Apotecio lecideíno; asca con ocho ascosporas alargadas, con cuatro septos transversales, hialina y de 60 × $3 \mu \mathrm{m}$; paráfisis con septos y sin ramificación. Talo K-. Habita en laderas con suelo arcilloso desnudo y matorral ralo.

M.E.: Coracayoc, 3800 m, 05 Ago. 01, A. Ramírez \& A. Cano 648c (USM); Marca, 3020 m, 07 oct. 01, A. Ramírez \& A. Cano 780b (USM).

Familia Pertusuariaceae Körb. EX Körb.

\section{Ochrolechia sp.}

Liquen crustáceo, marrón claro; saxícola. Apotecio circular, plano, sésil, diámetro de $1 \mathrm{~mm}$, borde talino y epitecio marrón claro. Talo con córtex superior, heterómero con clorobionte Protococcus sp. Apotecio lecanorino; asca con 8 ascosporas; ascospora ovalada sin septos, hialina y de 25,4 x 14,9 $\mu \mathrm{m}$; paráfisis con septos y anostomosándose. Talo K-. Habita en laderas rocosas con pajonal de puna.

M.E.: Huashta Cruz, 4000 - 4200 m, 16 may. 00, A. Ramírez \& A. Cano 244(USM). 
Familia Physciaceae Zahlbr

\section{Clave para diferenciar las especies de la familia Physciaceae}

1a. Talo crustáceo

1b. Talo folioso

2a. Talo de color gris

Buellia punctata

2b. Talo de color marrón

Buellia pullata

3a. Talo sin córtex inferior. Heterodermia podocarpa

3b. Talo con córtex inferior.

4

4a. Talo de color gris claro, córtex superior K + Physcia stellaris

4b. Talo de color gris oscuro, córtex superior K Phaeophyscia endococcinodes

\section{Buellia pullata Tuck.}

Liquen crustáceo areolado, marrón claro; saxícola. Apotecio circular, convexo, sésil, diámetro de $1 \mathrm{~mm}$, sin borde talino y epitecio negro. Talo con córtex, heterómero con clorobionte Trebouxia sp. Apotecio lecideíno; asca con ocho ascosporas elipsoidales, con un septo transversal, marrón y de $14,4 \times 6,72 \mu \mathrm{m}$; paráfisis con septos y sin ramificación. Talo K-. Habita en roquedales.

M.E.: Cerro Yanayco, 2400-3000 m, 01 nov. 00, A. Ramírez \& A. Cano 365 (USM).

\section{Buellia punctata (Hoffm.) Massal.}

Liquen crustáceo areolado con lóbulos, gris; saxícola. Apotecio circular, convexo, sésil, diámetro de 0,8 a $1 \mathrm{~mm}$, sin borde talino y epitecio negro. Talo con córtex, heterómero con clorobionte Trebouxia sp. Apotecio lecideíno; asca con 8 ascosporas elipsoidales con un septo transversal, marrón y de 14,4 x 4,8 $\mu \mathrm{m}$; paráfisis sin septo y ramificado. Talo K-. Habita en laderas rocosa-pedregosas con matorral.

M.E.: Cerro Yanaico, 2400-2650 m, 12 abr. 01, A. Ramírez \& A. Cano 404 (USM).

\section{Heterodermia podocarpa (Bél.) Awasthi}

Liquen folioso, gris; cortícola. Talo con cilios y rizinas. Apotecio circular, cóncavo, cortamente pedicelado, diámetro de 1 a $3 \mathrm{~mm}$, con borde talino y epitecio negro. Talo con córtex superior y sin córtex inferior, heterómero con clorobionte Trebouxia sp. Apotecio lecanorino; asca con ocho ascosporas elipsoidales con un septo transversal, marrón y de 14,4 x $3,17 \mu \mathrm{m}$; paráfisis con septo y ramificación. Talo $\mathrm{K}-$. Habita en matorrales.

M.E.: Cerro Nuna Riqoq, 3400 m, 17 may. 00, A. Ramírez \& A. Cano 228a (USM).

\section{Phaeophyscia endococcinodes (Poelt) Esslinger}

Liquen folioso, marrón; cortícola. Talo sin soredios y con rizinas simple. Apotecio circular, plano a cóncavo, sésil, diámetro de 0,8 a $1,7 \mathrm{~mm}$, con borde talino y epitecio marrón. Talo con córtex superior e inferior, heterómero con clorobionte Trebouxia sp. Apotecio lecanorino; asca con ocho ascosporas elipsoidales con un septo transversal, marrón y de 19,7 x 9,6 $\mu \mathrm{m}$; paráfisis con septos y ramificación. Talo K-. Habita en matorrales.

M.E.: Cerro Nuna Riqoq, 3400 m, 17 may. 00, A. Ramírez \& A. Cano 231 (USM); Pueblo Libre, 2560 m, 04 ago. 01, A. Ramírez \& A. Cano 615b (USM).

\section{Physcia stellaris (L.) Nyl.}

Liquen folioso, blanquecino; cortícola. Apotecio circular, plano, cortamente pedicelado, diámetro de $1,2 \mathrm{~mm}$, con borde talino y epitecio gris pruinoso. Talo con córtex superior e inferior, heterómero con clorobionte Trebouxia sp. Apotecio lecanorino; asca con ocho ascosporas elipsoidales con un septo transversal, marrón y de 16,4 x 7,7 $\mu \mathrm{m}$; paráfisis con septos y sin ramificación. Talo $\mathrm{K}+$ (amarillo). Habita en matorrales.

M.E.: Cerro Nuna Riqoq 3400 m, 17 may. 00, A. Ramírez \& A. Cano 228b (USM); Hoyada, 2800-3000 m, 07 oct. 01, A. Ramírez \& A. Cano 742 (USM). 
Familia Psoraceae Zahlbr.

\section{Psora icterica (Mont.) Müll. Arg.}

Liquen con escamas elongadas y márgenes ascendentes, verde claro; terrícola. Apotecio circular, convexo, sésil, diámetro de $0.5 \mathrm{~mm}$, sin borde talino y epitecio negro. Talo con córtex superior, heterómero con clorobionte Protococcus sp. Apoteciolecideíno, hipotecio marrón a oscuro, asca con ocho ascosporas elipsoidales sin septo, hialina y de $13 \times 5,76 \mu \mathrm{m}$; paráfisis con septo y sin ramificación. Talo K-. Habita en pajonales de puna y laderas con matorral.

M.E.: Huashta Cruz, 4000-4200 m, 16 may. 00, A. Ramírez \& A. Cano 212 (USM); Cerro Yanaico, 2400-3000 m, 01 nov. 00, A. Ramírez \& A. Cano 373 (USM).

\section{Familia Ramalinaceae Ag.}

\section{Ramalina celastri (Sprengel.) Krog \& Swinscow.}

Liquen fructicoso, verde oliva; cortícola. Apotecio circular, cóncavo, cortamente pedicelado, diámetro de 0,8 a $1 \mathrm{~mm}$, con borde talino y epitecio verde claro. Talo aplanado, sin soredios, sin isidios, apotecios marginales, sólido; con córtex, heterómero con clorobionte Protococcus sp. Apotecio lecanorino; asca con ocho ascosporas elipsoidales con un septo transversal, hialina y de 3,8 x $1 \mu \mathrm{m}$; paráfisis con septo y sin ramificación. Talo K-. Habita en pajonales puna y matorrales.

M.E.: Cerro Riqoq, 3400 m, 17 may. 00, A. Ramírez \& A. Cano 251 (USM); Coracayoc, 3400-3940 m, 05 ago. 01, A. Ramírez \& A. Cano 625 (USM).

Determinado: Dr. Kashiwadani H.

Familia Rhizocarpaceae M. Choisy eX HAFELLNER

30. Rhizocarpon geographicum (L). Lam \& Dc.
Liquen crustáceo, talo amarillo y apotecio negro; saxícola. Apotecio irregular, plano, inmerso, diámetro de 0,2 a $0,8 \mathrm{~mm}$, sin borde talino y epitecio negro. Talo con córtex superior, heterómero con clorobionte Trebouxia sp. Apotecio lecideíno; asca con ocho ascosporas elipsoidales con muchos septos, marrón y de 42,9 x 21,1 $\mu \mathrm{m}$; paráfisis con septos y sin ramificación. Talo K- y médula $\mathrm{K}-$. Habita en roquedales con matorral.

M.E.: Huashta Cruz, 4000-4200 m, 16 may. 00, A. Ramírez \& A. Cano 242 (USM); Antircán, 3450 m, 08 ago. 01, A. Ramírez \& A. Cano 681 (USM).

Familia Teloschistaceae Zahlbr.

\section{Clave para diferenciar las especies de la familia Teloschistaceae}

1a. Talo crustáceo 2

1b. Talo fructicoso Teloschistes chrysopthalmus

2a. Talo crustáceo sin márgenes lobados Caloplaca cinnabarina

2b. Talo crustáceo con márgenes lobados 3

3a. Talo con márgenes cortos Caloplaca saxícola

3b. Talo con márgenes largos Caloplaca sp.

\section{Caloplaca cinnabarina (Ach.)} Zahlbr.

Liquen crustáceo areolado, anaranjado; saxícola. Apotecio circular, plano, sésil, diámetro de 0,5 a $1 \mathrm{~mm}$, con borde talino y epitecio anaranjado. Talo con córtex superior, heterómero con clorobionte Trebouxia sp. Apotecio lecanorino; asca con ocho ascosporas elipsoidales, polarilocular, hialina, de 10,8 x 5,4 $\mu \mathrm{m}$; paráfisis con septos y sin ramificación. Talo $\mathrm{K}+$ (púrpura). Habita en roquedales.

M.E.: Huashta Cruz, 4000-4200 m, 16 may. 00, A. Ramírez \& A. Cano 243 (USM).

\section{Caloplaca saxícola (Hoffm.) Nordin}

Liquen crustáceo areolado en el centro y hacia los márgenes formando lóbulos; anaran- 
jado; saxícola. Apotecio circular, plano, sésil, diámetro de $5 \mathrm{~mm}$, con borde talino y epitecio anaranjado. Talo con córtex superior, heterómero con clorobionte Trebouxia sp. Apotecio lecanorino; asca con ocho ascosporas elipsoidales, polarilocular, hialina y de 9 x $5 \mu \mathrm{m}$; paráfisis con septos y ramificación en el ápice. Talo K + (púrpura). Habita en laderas pedregosas y cumbres rocosas. Fig. $2 \mathrm{~b}$.

M.E.: Huashta Cruz, 3500 m, 26 may. 01, A. Ramírez \& A. Cano 486 (USM); Angüel Pampa, 2550m, 10 ago.01, A. Ramírez 710,704 (USM); Coiroqsho, 2900-3220 m, 08 oct. 01, A. Ramírez \& A. Cano 803 (USM); Tocash, 2320 m, 09 oct. 01, A. Ramírez \& A. Cano 818 (USM); Pueblo Libre, 04 ago. 01, 2500 m, A. Ramírez \& A. Cano 613 (USM).

\section{Caloplaca sp.}

Liquen crustáceo areolado en el centro y hacia los márgenes ramificándose largamente y formando lóbulos; anaranjado rojizo; saxícola. Apotecio ausente. Talo con córtex superior, heterómero con clorobionte Trebouxia sp. Talo K+ (púrpura). Hábitat: Ladera rocosa con matorral.

M.E.: Cerro Yanaico, 2400-2650 m, 12 may. 01, A. Ramírez \& A. Cano 416 (USM).

\section{Teloschistes chrysophthalmus (L.) Th. Fr.}

Liquen fructicoso de $20 \mathrm{~mm}$, anaranjado; cortícola. Apotecio circular, cóncavo a plano, sésil, diámetro de 1 a 2,5 mm, con cilios, con borde talino y epitecio anaranjado. Talo sin soredio, sin cilios, sólido, con córtex, heterómero con clorobionte Trebouxia sp. Apotecio lecanorino; asca con ocho ascosporas elipsoidales, polarilocular, hialina y de 13,4 x 6,7 $\mu \mathrm{m}$; paráfisis con septos y ramificación en el ápice. Talo K+ (púrpura). Habita en laderas con matorral. (Fig. 3e)

M.E.: Marca, 3020 m, 07 oct. 01, A. Ramírez \& A. Cano 771 (USM).
Familia Thelephoraceae (Meruliaceae P. KARSTEN)

\section{Clave para diferenciar las especies del género Dictyonema}

1a. Hifa con asa y cianobionte Scytonema

Dictyonema ircipium

1b. Hifa sin asa y cianobionte Chroococcus

Dictyonema glabratum

\section{Dictyonema ircipium Mont.}

Liquen folioso, gris; terrícola. Sin presencia de basidiosporas. Talo con lóbulos semicirculares y reniformes, pequeños de $1 \mathrm{~cm}$, involuto y en la cara inferior con tomento. Talo sin córtex superior e inferior, hifas con asa y cianobionte Scytonema sp. Talo K-. Habita en pajonales de puna.

M.E.: Huashta Cruz, 4090 m, 16 may. 00, A. Ramírez \& A. Cano 218 (USM).

36. Dictyonema glabratum (Spreng.) D. Hawksw.

Liquen folioso, blanco; muscícola. Sin presencia de basidiospora. Talo con lóbulos semicirculares y reniformes, grandes de 1,5 $\mathrm{cm}$, involuto y en la cara inferior con tomento. Talo sin córtex superior e inferior, heterómero, hifas sin asa y cianobionte Chroococcus sp. Talo K-. Habita en pajonales de puna y matorrales. (Fig. 2d)

M.E.: Huashta Cruz, 4090 m, 16 may. 00, A. Ramírez \& A. Cano 219 (USM); Cerro Yanaico, 3100 m, 12 abr. 01, A. Ramírez \& A. Cano 406 (USM); Antircán, 3450 m, 08 ago. 01, A. Ramírez \& A. Cano 684 (USM).

Familia Umbilicariaceae Chev.

\section{Umbilicaria dichroa Nyl.}

Liquen folioso con ombligo; cara superior gris o marrón e inferior negra, con rizinas simples y dicotómicas de color negro o marrón; saxícola. Apotecio circular, plano, pedicelado de 0,8 a $3 \mathrm{~mm}$, diámetro de $1,5 \mathrm{~mm}$, sin borde 
talino y epitecio negro. Talo con córtex superior e inferior, heterómero con clorobionte Trebouxia sp. Apotecio lecideíno; asca con ocho ascosporas elipsoidales sin septo, hialina y de 17,2 x 9,6 $\mu \mathrm{m}$; paráfisis con septo y sin ramificación. Talo $\mathrm{K}-$. Habita en roquedales de altura.

M.E: Huashta Cruz, 4090 m, 16 may. 00, A. Ramírez \& A. Cano 211 (USM). Huashta Cruz, 3800 m, 13 abr. 01, A. Ramírez \& A. Cano 436 (USM).

Determinado por: Dr. Hetsmark G.

\section{Grupo Imperfecto}

\section{Thamnolia vermicularis (Sw.) Schaer.}

Liquen fructicoso, blanco; muscícola. Apotecio ausente. Talo con córtex, heterómero con clorobionte Trebouxia sp. y hueco. El Talo $\mathrm{K}-$. Habita en pajonales de puna.

M.E.: Huashta Cruz, 4090 m, 16 may.00, A. Ramírez \& A. Cano 216 (USM). Fig. 2 f.

\section{Discusión y conclusión}

Los líquenes en Ancash son muy poco conocidos Algunas publicaciones (Herrera, 1941; Soukup, 1965; Tokumine, 1985; Walker, 1985; Elix \& Nash, 1992; Nash et al., 1995; Tovar , 1996; han reportado la presencia no más que 19 especies de líquenes en el departamento de Ancash. La familia Arthoniaceae y los géneros Acarospora, Arthonia, Buellia, Candelaria, Cetrariastrum, Flavoparmelia, Pseudevernia, Phaeophyscia, Ochrolechia, Placomaronea y Psora son reportados por primera vez en el departamento de Ancash. De las 38 especies que reportamos en el presente trabajo, 34 son primer reporte para el departamento de Ancash y solamente Everniopsis trulla, Dictyonema glabratum, Usnea durietzii y Psiloparmelia distincta fueron reportadas anteriormente.

Hasta ahora son reportados alrededor de 390 especies para la liquenobiota del Perú
(Feuerer, 2005 y otras referencias bibliográficas). En el presente trabajo se da reporta por primera vez para el Perú 15 especies: Arthonia lapidicola, Arthonia rupicola, Candelaria concolor, Cetrariastrum sp. Lecidea auriculata, Lecidea vorticosa, Flavoparmelia scabrosina, Pseudevernia sp., Ochrolechia sp., Buellia pullata, Buellia punctata, Caloplaca cinnabarina, Dictyonema ircipium, Umbilicaria dichroa y Xanthoparmelia conspersa.

\section{Agradecimientos}

Los autores agradecen por el apoyo económico, National Geographic Society a través del proyecto «Plant Diversity and Endemism in Peru`s Central Highlands»; al Fondo de Desarrollo Universitario (FEDU) de la Universidad Nacional Mayor San Marcos con el proyecto «Diversidad florística y endemismo en las Cordilleras Blanca y Negra (Ancash)». El primer autor agradece el apoyo de la $\mathrm{Fa}-$ cultad de Ciencias Biológicas de la UNMSM, por la beca otorgada para la ejecución de la tesis para optar el título profesional de Biólogo. También nuestra profunda gratitud con los especialistas extranjeros: Harrie Sipman, Roland Moberg, Teuvo Ahti, David Galloway, Hiroshi Kashiwadani, Thom Nash, John Elix, Thorsten Lumbsch, Nora Wirtz y Geir Hestmark, por su valiosa colaboración con las determinaciones y con literatura especializada. Quedamos igualmente reconocidos con nuestros colegas: Magda Chanco, Blanca León, María I. La Torre, José Roque y Erick Rodríguez, por su colaboración en alguna etapa del estudio y en la, preparación del presente trabajo.

\section{Literatura Citada}

Ahti, T. \& H. Kashiwadani. 1984. The lichen genera Cladia, Cladina and Cladonia in Southern Chile. Editor Inoue Hiroshi. Pp. 125 - 151.

Almborn, I. 1992. Some overlooked or misidentified species of Teloschistes from South America and Key to the South- American species. Nord. J. Bot. 12: 361 - 264. Copenhagen.

Barreno, E. 1998. Hongos Simbiontes. Botánica. España. McGraw-Hill. Pp. 309-335. 
Brodo I., Durand S. \& S. Sharnoff. 2001. Lichens of North America. Yale University Press, New Haven and London. Pp 795.

Coutino, B. 1986. Manual de Herbario México. Consejo Nacional de Flora de Méjico. Pp. 65 - 75.

Elix J. 1994. Flavoparmelia. Flora de Australia Lichens: Pp 39 - 49 .

Elix J. \& Nash T. 1992. A synopsis of Lichen Genus Psiloparmelia (Ascomycotina, Parmeliaceae). The Bryologist 95 (4), 1992. Pp. 377 - 391.

Ferreyra, R. 1986. Gran geografía del Perú - Naturaleza y Hombre. Volumen II. Editorial Juan Mejia Baca, Ediciones Manfer. Impreso en España. Grafos S.A. Barcelona. p 197.

Feuerer, T. 2005. Checklist of Peruvian lichens and lichenicolous fungi of Peru. Versión 1 April 2002. http://www.biologie.uni-hamburg.de/ checklists/peru 1.htm

Fink B. 1960. The lichen flora of United States of America. Copyright $\mathrm{c}$ by the University of Michigan. $426 \mathrm{pp}$.

Galloway, D. 1985. Flora of New Zealand Lichens. Manaaki Whenua Press. 662 pp.

Galloway, D. 1994. Studies on the lichen genus Sticta (Schreber) Ach. I. Southern South American species. - Lichenologist 26: 223 - 282.

Herrera, F. 1941. Sinopsis de la flora del Cusco. Tomo I. Parte Sistemática. Publicado bajo los auspicios del Supremo Gobierno. Pp. 50 - 64.

Herrera, T. \& Ulloa, M. 1990. El Reino de los Hongos. México. Fondo de Cultura Económica. Pp. $343-363$.

Kashiwadani, H. 1987. Peruvian Species of Ramalina (Lichens). Studies on Crytogams in Southern Peru. (ed. H. Inoue). Tokai University Press. Pp. 129 -144.

Moberg, R. 1990. The lichen genus Physcia in Central and South America. - Nord. J. Bot. 10: 319 342 .
Moberg, R. 1993. The lichen genus Phaeophyscia in South America with special reference to Andean species. Opera Bot. 121: 281-284.

Nash T., C. Gries \& J. Elix. 1995. A Revisión of the Lichen Genus Xanthoparmelia in South America. Berlin - Stuttgart. 157 pp.

Parmastro, E. 1987. The Genus Dictyonema. Nova. Hedwigia 29. Pp. 99-42.

Ramírez, R. 1969. Líquenes de las Lomas de La Provincia de Trujillo. Separata de la Revista de la facultad de Ciencias Biológicas. Vol II, $\mathrm{n}^{\circ} 1$. Trujillo. Pp. 55-70.

Sato, M. 1968. Two New Varieties of Thamnolia from South America. Bryologist. Vol. 71. Pp. 49 50.

Sipman, H. 1999. Identification Key and literature guide to the genera of Lichinized Fungi (Lichens) in the Neotropics. http://www.bgbm.fuberlin.de/sipman/keys/neokeyA.htm.

Soukup, J. 1965. Lista de Líquenes del Perú. Lima. Biota. Pp. 28-45.

Tokumine, J. 1985. Líquenes del Herbario de San Marcos. Informe Practicas pre-profesional (monografía). Programa Académico de Ciencias Biológicas. Universidad Nacional Mayor de San Marcos. Lima. 15 pp.

Tovar, D. \& Aguinaga R., 1994. Líquenes como indicadores de la contaminación atmosférica en Lima metropolitana. Revista de química. Vol VIII. n², diciembre. Lima. Pp. 135-152.

Tovar, D. 1996.Líquenes fijadores de nitrógeno y sus ficobiontes en cultivo. Consejo Nacional de Ciencia y Tecnologia - Perú. SERIE Ciencias. Editorial Hozlo S.R.L. 157 pp.

Vitikainen O. 1998. A Preliminary Key to Peltigera in the Neotropics. En Marcelli \& M.R.D. Seaward (Eds). Lichenology in Latin America: history, current knowlegde and applications. Pp 117-128. CETESB. São Paulo.

Walker F. 1985. The lichen genus Usnea subgenus Neuropogon. Bolletin of Brithish Museum. Natural. History. (Botany.) 13 (1): $1-130$. 